\title{
Neurobiology of Pediatric Anxiety Disorders
}

\author{
Jeffrey R. Strawn • Kelli C. Dominick • Luis R. Patino • \\ Christopher D. Doyle • Lara S. Picard • K. Luan Phan
}

Published online: 19 June 2014

(C) Springer International Publishing AG 2014

\begin{abstract}
While the fear-based anxiety disorders (i.e., generalized anxiety disorder, social phobia and separation anxiety disorder) are among the most common psychiatric conditions in children and adolescents, only recently has an integrated understanding of the neurobiology of these disorders developed. In this regard, both structural and functional neuroimaging studies have demonstrated neuroanatomic and functional abnormalities within the amygdala and prefrontal cortex in youth with fear-based anxiety disorders, and have also suggested altered functional connectivity among components of the anterior limbic network (ALN), as well as alterations in neurochemistry within the anterior cingulate cortex. Additionally, several prefrontal structures and regions (e.g., medial prefrontal cortex) appear to be dysregulated in youth who are at risk of developing anxiety disorders (e.g., youth with inhibited temperament, behavioral inhibition, etc.). Finally, emerging data raise the possibility that functional activity within these amygdala-prefrontal networks may be affected
\end{abstract}

J. R. Strawn · K. C. Dominick · L. R. Patino · C. D. Doyle
Department of Psychiatry and Behavioral Neuroscience, University
of Cincinnati, College of Medicine, Cincinnati, OH 45219, USA

J. R. Strawn $(\bowtie) \cdot$ K. C. Dominick

Department of Pediatrics, Division of Child \& Adolescent Psychiatry, Cincinnati Children's Hospital Medical Center, Box 670559, Cincinnati, OH 45267-0559, USA

e-mail: strawnjr@uc.edu

L. S. Picard

James L. Winkle College of Pharmacy, University of Cincinnati, Cincinnati, OH, USA

\section{K. L. Phan}

Departments of Psychiatry, Psychology and Anatomy and Cell

Biology, University of Illinois at Chicago, Cincinnati, OH, USA

K. L. Phan

Mental Health Service Line, Jesse Brown VA Medical Center,

Chicago, IL, USA by successful psychopharmacologic and psychotherapeutic treatment and may predict outcome.

Keywords fMRI $\cdot{ }^{1} \mathrm{H}$ MRS $\cdot$ Anxiety disorders $\cdot$ Separation anxiety disorder (SAD) $\cdot$ Social phobia (SoP) $\cdot$ Generalized anxiety disorder (GAD)

\section{Introduction}

First described in pediatric patients more than a century ago [1], anxiety disorders are among the most prevalent disorders in children and adolescents, with prevalence rates reaching $10 \%[2 \cdot, 3-5]$. Moreover, anxiety disorders - in children and adolescents - represent chronic, relapsing conditions [2•] and are associated with substantial morbidity, including an increased risk of suicide attempts [6], secondary mood disorders $[7,8]$, and co-occurring anxiety disorders $[7,8]$. Yet, despite their profound public health significance and associated morbidity, only recently has the structural and functional neuroanatomy as well as the neurochemistry of these disorders been systematically examined in youth.

Over the last decade, accumulating evidence suggests that there are specific alterations within central fear networks in anxiety disorders in youth [9-11]. This includes the anterior limbic network (ALN), which involves connections between the amygdala, medial prefrontal cortex (Brodmann area [BA] 10/11), rostral insula, subgenual/rostral anterior cingulate cortex (ACC, BA 25, BA 24/32), (Fig. 1a), ventrolateral prefrontal cortex (VLPFC, BA 10/47) (Fig. 1b), and dorsolateral prefrontal cortex. This network has been extensively studied in children and adolescents with generalized anxiety disorder (GAD), social phobia (SoP) and separation anxiety disorder (SAD), and as a consequence of these studies, a nascent understanding of (1) the neurostructural and neurofunctional basis of pediatric anxiety disorders, as well as (2) the 


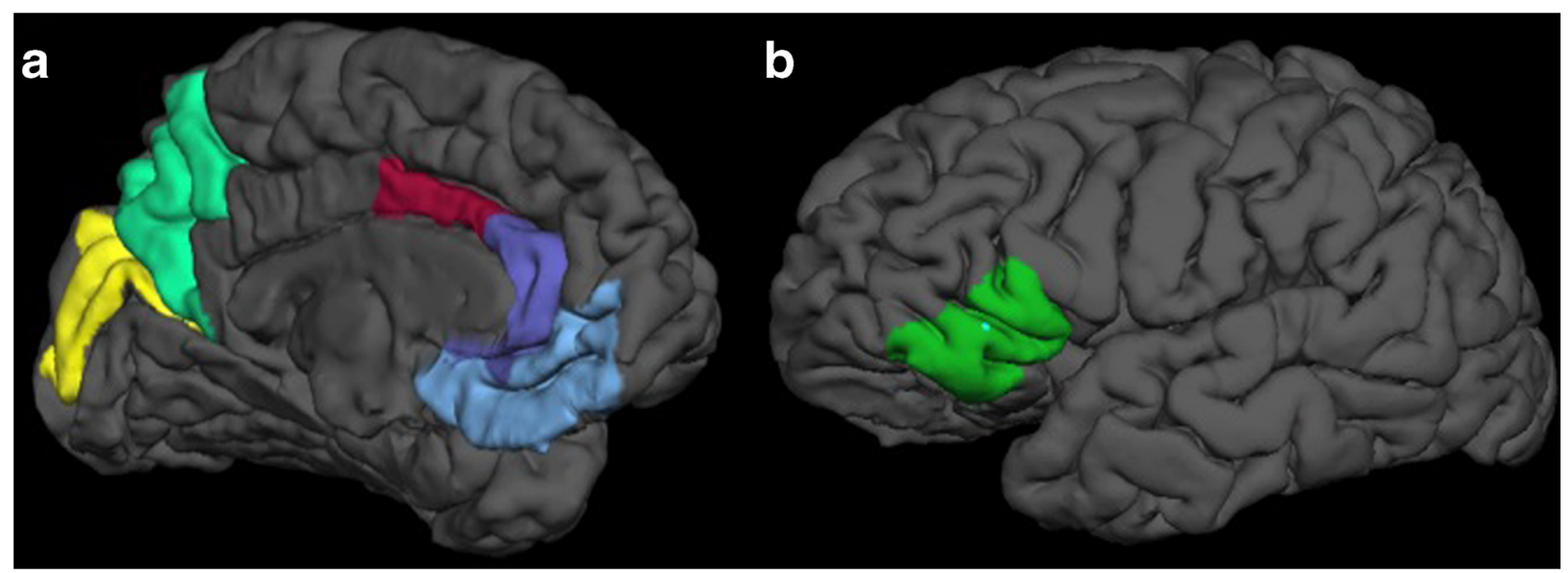

Fig 1 Structures and regions implicated in pediatric patients with anxiety disorders and those who are high risk of developing anxiety disorders. The cuneus (yellow), precuneus (light green), dorsal anterior cingulate (red), pre/

neurobiologic basis of risk for developing these disorders, has developed. Herein, we seek to systematically review and integrate neurostrucutural, neurofunctional and neurochemical derangements within these systems in pediatric patients who are at risk for developing anxiety disorders (i.e., those with temperaments which predispose them to develop anxiety disorders such as behavioral inhibition), as well as those who have developed a pediatric anxiety triad disorder: GAD, SoP and/or SAD.

\section{The Neurofunctional Basis of Generalized Anxiety Disorder}

The most notable and frequently implicated structure in pediatric anxiety disorders, including GAD, is the amygdala. Residing deep within the limbic system, this subcortical structure is critical to the generation of the central the fear response. Moreover, the amygdala is comprised of multiple nuclei that are reciprocally connected to the hypothalamus, hippocampus, and neocortex-structures which, as discussed below, have been implicated in the pathophysiology of anxiety disorders. To date, nearly all $(n=9)$ studies of pediatric patients with GAD have demonstrated increased activation of the amygdala (for review see [12]), although it is important to note that these have primarily involved the presentation of fearful faces in the context of facial affect probes. Specifically, McClure and colleagues [13] observed increased right amygdala activation in adolescents with GAD when viewing fearful faces, while bilateral activation was observed in the context of a probe detection tasks involving paired emotional faces [13]. However, when tasks involve attentional processing of nonemotional stimuli, differences in amygdala activation are not observed in adolescents with GAD [10]. Finally, it is noteworthy that in a sample of anxious youth, most of whom met Diagnostic and Statistical Manual of Mental Disorders, Fourth subgenual anterior cingulate (purple) and ventromedial prefrontal cortex (light blue) are show medially in a, while the ventrolateral prefrontal cortex (VLPFC, lime green) is shown in the left lateral view in $\mathbf{b}$

Edition (DSM-IV) criteria for GAD, baseline levels of amygdala activation was negatively associated with symptomatic improvement following treatment with either the selective serotonin reuptake inhibitor fluoxetine or cognitive behavioral therapy [14].

In addition to increased amygdala activation, adolescents with GAD exhibit dysfunction within amygdala-based intrinsic functional connectivity networks, which includes connectivity between the amygdala and regions in medial prefrontal cortex, insula, and cerebellum. In this regard, amygdala-insula connectivity and amygdala-superior temporal gyrus connectivity increases with increased anxiety burden in adolescents with GAD [15•]. Additionally, in adolescents with GAD the activation of amygdala, ventral prefrontal cortex, and ACC are highly correlated during the viewing fearful faces as compared to healthy subjects [14]. Seed-based connectivity analyses suggest increased connectivity between the amygdala and insula as well as the amygdala and posterior cingulate cortex in patients with GAD compared to healthy subjects, whereas there is greater negative connectivity between the amygdala and precuneus in patients compared to healthy subjects [14]. Finally, steady state connectivity analyses of adolescents with GAD are consistent with the findings above with regard to increased connectivity between the amygdala and the posterior cingulate cortex and altered amygdala-precuneus connectivity in adolescents with GAD during continuous processing tasks [10] - an interesting finding, given that the posterior cingulate and precuneus/cuneus are intimately involved in mentalization and theory of mind processes as well as self-referential cognition; functions that are presumably disrupted in youth with GAD.

Beyond the amygdala, the VLPFC is frequently implicated in fMRI studies of youth with GAD. Of note, the VLPFC modulates amygdala activity and plays a pivotal role in extinction in the context of fear conditioning and responds in tandem with the amygdala to emotional probes. Thus, given the role of the VLPFC in modulating amygdala activity and 
fear learning — a process which is fundamentally impaired in youth with GAD [16] - it is not surprising that dysfunctional activity within the VLPFC and dysfunctional connectivity to this region is frequently observed in pediatric patients with GAD. In fact, to date, at least three fMRI studies that focused specifically on patients with GAD have demonstrated abnormal activation within this structure $[17,18,10]$. However, it is likely that the role of the VLPFC in pediatric GAD is complex, in that - while this region is hyperactivated in youth with GAD - activation is inversely related to the severity of anxiety symptoms [10]. This complex relationship between activation and anxiety within this structure suggests that the VLPFC may play a compensatory role in youth with GAD. As such, one small treatment study involving administration of the SSRI fluoxetine or cognitive-behavioral therapy (CBT) demonstrated treatment-associated increases in activity within this region in adolescents with GAD [19].

Finally, regarding connectivity of the VLPFC in adolescents with GAD, McClure and colleagues [14] found that VLPFC activity correlated with amygdala and ACC activation in youth with GAD. Additionally, in this study, increased negative connectivity was observed between the right VLPFC and amygdala in youth with GAD (compared to healthy subjects) [18]. Finally, we recently observed increased connectivity between the VLPFC and the medial prefrontal cortex in adolescents with GAD - a structure that subserves both self-referential processing and interoception [10].

In addition to the amygdala and VLPFC, the cingulate cortex (Fig. 1a) - which forms the superior boundary of the limbic system - is intimately involved in affect and motivation, as well as pain processing and cognitive control (for review see $[20 \bullet, 21]$. The cingulate, in particular the anterior cingulate, cortex has regularly been implicated in several of the fear-based anxiety disorders in youth, in particular, GAD. In this regard, McClure and colleagues [14] demonstrated that youth with GAD who were attending to their own subjective fear during the performance of a facial affect task have increased activation of the anterior cingulate cortex. Additionally, a recent proton $\left({ }^{1} \mathrm{H}\right)$ spectroscopy study of adolescents with GAD demonstrated that the severity of anxiety symptoms correlated with increased glutamatergic tone in the pregenual anterior cingulate [22].

\section{The Neurostructural Basis of Generalized Anxiety Disorder}

In addition to accumulating data regarding functional activity and functional connectivity of GAD in adolescents, several studies have examined the neurostructural basis of this disorder. Overall, the literature suggests that abnormalities in neural structures that subserve threat appraisal, modulation of fear responses, attachment, and mentalization are present early in the course of the disorder. Amygdala volumes are increased in adolescents with GAD when examined with manual tracing methods [23], although voxel-based morphometry-based studies have observed decreased left amygdala volumes in adolescents with mixed anxiety disorders, of whom nearly $80 \%$ met criteria for GAD [24, 23]. Additionally, in this voxel-based morphometry study, gray matter volumes were increased in the superior temporal gyrus, a structure which shares numerous connections with the amygdala [24]. Moreover, we have demonstrated decreased gray matter volumes in in the right precuneus and right precentral gyrus as well as decreased gray matter volumes in the left orbital gyrus and posterior cingulate in un-medicated adolescents with GAD, [25].

Finally, diffusor tensor imaging and voxel-wise TractBased Spatial Statistics (TBSS) analysis of white matter architecture in adolescents with GAD reveals decreased fractional anisotropy (FA), a measure of white matter integrity, in the bilateral uncinate fasciculus, inferior fronto-occipital fasciculus, as well as the inferior longitudinal fasciculus, and corona radiata [26].

\section{The Neurofunctional Basis of Social Phobia}

Many functional imaging studies in pediatric patients with social phobia have - necessarily_-involved tasks with social judgments in an effort to probe the aspects of social phobia which may be neurophysiologically distinct from the structures which are putatively involved in the other fear-based anxiety disorders. Studies have demonstrated abnormalities in those structures implicated in other fear-based disorders, namely the amygdala and its cortical connections, as well as altered striatal activity in youth with SoP. In one such study involving adolescents with SoP who are anticipating social feedback, caudate and putamen activation is increased compared to adolescents who are healthy and those who have generalized anxiety disorder [27]. Additionally, as adolescents with social phobia await feedback from individuals with whom they might prefer not to interact (vs. those with whom they would interact), activation in the amygdala is increased and is associated with increased amygdala-VLPFC connectivity in anxious vs. healthy adolescents in response to these stimuli, suggesting that "anticipating social evaluation from negatively perceived peers modulates amygdala and VLPFC engagement differentially in anxious and healthy adolescents" [28].

\section{The Neurofunctional Basis of Separation Anxiety Disorder}

To date, no studies have specifically examined youth with a primary diagnosis of separation anxiety disorder. However, in 
a study of youth with social anxiety which utilized a facial affect probe, bilateral amygdala activity correlated with the degree of separation anxiety [29], suggesting that the amygdala may be implicated in the pathophysiology of separation anxiety disorder as well.

\section{The Neurofunctional Basis of Mixed Anxiety Disorders}

Studies of "mixed anxiety disorders" have frequently included patients with the triad of GAD, SoP and separation anxiety disorder, given the high syndromic overlap of these conditions and frequent comorbidity. In general, these studies have focused on anterior limbic circuitry described previously in patients with GAD and social phobia. Price and colleagues [30] noted that,while both youth with anxiety disorders and healthy comparison subjects demonstrated "a neural pattern consistent with successful disengagement of threat reactivity in the [rostral-dorsal] ACC", youth with anxiety did not disengage with regard to bilateral parahippocampal/hippocampal regions when avoiding threat, suggesting poorer integration of cortical and limbic components of threat appraisal. Additionally, this poorer integration of cortical and lower-limbic structures may be mediated by decreased functional connectivity between the dorsal anterior cingulate and left parahippocampus/hippocampus, in which decreased connectivity was correlated with increased anxiety [30]. Fitzgerald and colleagues [31] have demonstrated that, during error detection, the dorsolateral prefrontal cortex is hypoactive (compared to healthy subjects) in youth with non-obsessivecompulsive disorder (OCD) anxiety. Moreover, it is of interest that patients with mixed anxiety disorders and co-occurring major depressive disorder (MDD) exhibit similar increases in bilateral amygdala activation in response to fearful face processing compared to healthy comparison subjects; however, adolescents with mixed anxiety disorders compared to with MDD (without co-occurring anxiety disorders) exhibit differential amygdalar responses during passive viewing of fearful faces [32]. Finally, a recent pilot study of youth with mixed anxiety disorders $(N=10)$ - who were at genetic risk of developing bipolar disorder - demonstrated that mindfulness-based cognitive therapy was associated with increased activation of the bilateral insula, lentiform nucleus, thalamus and the left anterior cingulate during the viewing emotional stimuli [33].

\section{The Neurostructural Basis of Mixed Anxiety Disorders}

Regarding neurostructural studies of adolescents with mixed anxiety disorders, Mueller and colleagues [34], using a regionof-interest (ROI) approach (ROIs: amygdala, hippocampus, insula and ACC), noted decreased gray matter volumes in adolescents with anxiety in the amygdala and right anterior hippocampus, and noted increased gray matter volume in the right insula. In this study, a group-by-genotype effect for a Val ${ }^{66}$ Met polymorphism in the brain-derived neurotrophic factor gene was observed in the insula and ACC [34]. Additionally, in a sample of 38 youth with mixed anxiety disorders and 27 healthy comparison subjects, we observed greater gray matter volumes in the left cingulate gyrus compared to healthy subjects' decreased gray matter volume in the left inferior frontal gyrus (VLPFC), as well as the left postcentral gyrus and left precuneus [25]. Moreover, in this study, to elucidate any contribution of SoP symptoms to neuroanatomic differences in regions where gray matter volume differences were detected between anxious and healthy youth, we noted no relationship between social phobia symptoms and gray matter volumes [35]. Additionally, we have observed decreased gray matter volumes in the dorsolateral prefrontal cortex in youth with co-occurring anxiety disorders and major depressive disorder compared to adolescents with major depressive disorder and no co-occurring anxiety disorders [36]. Taken in consideration with the neurofunctional data [32], this would suggest both shared and distinct neurofunctional abnormalities along the depression-anxiety diathesis.

\section{The Neurofunctional Basis of Risk in Patients Who Are Predisposed to Developing Anxiety Disorders}

Recently, several studies have examined the neurophysiology of children who are at risk for developing anxiety disorders, yet do not have anxiety disorders per se. In general, these studies have focused on younger children with anxious temperament and behavioral inhibition, both known to increase the likelihood of developing an anxiety disorder later in life [37]. These studies have shown abnormalities in the same regions seen in studies of youth with the triad of anxiety disorder discussed above. Overall, there is increased activation of the amygdala, abnormalities in cortical regions of the ALN, and as with SoP, some indication of striatal involvement.

Blackford and colleagues [38•] have suggested that dynamic responses to emotional stimuli may differ in studies of youth with inhibited temperament, a finding which has implications for fear learning (and extinction). Specifically, in individuals with inhibited temperament (e.g., those who are shy, inhibited and slow-to-warm-up), habituation of the amygdala and hippocampus to neutral faces varied as a function of the degree of inhibited temperament, wherein those individuals with inhibited temperament failed to exhibit habituation across serially presented faces [38•]. Additionally, in this sample of individuals with inhibited temperament, amygdala responses remain elevated when viewing both novel and "recently familiarized faces," whereas individuals without inhibited temperament only exhibit increased amygdala 
activity in response to novel faces [39]. Inhibited adolescents (compared to non-inhibited adolescents) also exhibit increased amygdala activation to fearful faces [40]. Moreover, in healthy youth who have high levels of trait anxiety, amygdala activity during the viewing of fearful faces correlates with "social dimensions of anxiety, including peer rejection, humiliation, performing in public, and being separated from loved ones," but does not correlate with nonsocial dimensions of anxiety [29]. Not only is the amygdala itself implicated in behavioral inhibition (BI), but the fronto-amygdala network as well. A recent study using an attention-bias task with angry and neutral faces found that youth with BI have altered connectivity between the amygdala and the dorsolateral prefrontal cortex or anterior insula [41], specifically a greater negative connectivity was seen between these regions in BI. In addition, they found that the amygdala-insula connectivity was related to young adult internalizing symptoms. Recently, a study of youth with high levels of trait anxiety, which utilized a paradigm to assess attention to threat, found that trait anxiety correlated with attention bias towards angry faces. In addition, trait anxiety positively predicted increased activation of the dorsolateral prefrontal cortex (PFC) during attention bias towards angry faces and - consistent with studies in youth with GAD - trait anxiety was associated with increased activation in the VLPFC [42]. In another recent study of young adults who exhibited behavioral inhibition as children (and who have dysregulated attention and affective processing), striatal activation as well as dorsolateral prefrontal cortex and anterior cingulate activation were observed relative to youth who were not characterized as behaviorally inhibited, despite the fact that both groups exhibited similar behavioral responses to a high attention to threatening faces task [43].

\section{Conclusion}

The extant literature concerning functional neuroimaging studies described herein suggests that pediatric patients with anxiety have dysfunction within a myriad of amygdala-based networks throughout the prefrontal cortex, and these studies have also increasingly implicated posterior regions, including the precuneus and cuneus. Moreover, functional abnormalities in these regions and in connectivity among these regions are present in youth and young adults who are at risk for developing anxiety disorders, suggesting that functional deficits may precede the disorders themselves. Additionally, accumulating data suggest that successful psychopharmacologic and psychotherapeutic interventions impact activity within these amygdala-based networks, and in some cases, activity within components of these fear-based networks may predict treatment response, although the treatment-related changes in functional activity within these circuits and the durability of these changes with regard to underlying functional or structural abnormalities observed in youth with anxiety disorders remains unknown. Moreover, neuroimaging data related to developmental trajectories of the anxiety disorders are brutally lacking, and there are limited data linking neuroimaging findings with symptom severity and functional outcomes (e.g., school performance, relational capacity with peers, etc.). Similarly, with increased interest in research domain criteria (RDoC), it is important to note that there are limited data related to neurofunctional and neurostructural commonalities among fear-based disorders in youth. Importantly, such studies in the pediatric population may yield 'brain targets' for early intervention and prevention of subsequent comorbidities (e.g., depression, other anxiety disorders, etc.).

\section{Compliance with Ethics Guidelines}

Conflict of Interest Jeffrey R. Strawn has received research support from Eli Lilly, Shire, Lundbeck and from the American Academy of Child \& Adolescent Psychiatry. Kelli C. Dominick and Lara S. Picard as well as Christopher D. Doyle report no biomedical conflicts of interest. K. Luan Phan has received funding from the National Institutes of Mental Health and from the Department of Veterans' Affairs

Human and Animal Rights and Informed Consent This article does not contain any studies with human or animal subjects performed by any of the authors.

\section{References}

Papers of particular interest, published recently, have been highlighted as:

- Of importance

1. Freud S. Analysis of Phobia in a five-year-old boy. In: Strachey J, editor. The Standard Edition of the complete Psychological Works of Sigmund Freud vol 10. London: Hogarth Press; 1909/1955. p. 3147.

2. Beesdo K, Pine DS, Lieb R, Wittchen HU. Incidence and risk patterns of anxiety and depressive disorders and categorization of generalized anxiety disorder. Arch Gen Psychiatry. 2010;67(1):4757. doi:10.1001/archgenpsychiatry.2009.177. This is a large, prospective examination of the risk factors and prevalence of anxiety disorders in youth.

3. Merikangas KR, He JP, Burstein M, Swanson SA, Avenevoli S, Cui L, et al. Lifetime prevalence of mental disorders in U.S. adolescents: results from the National Comorbidity Survey ReplicationAdolescent Supplement (NCS-A). J Am Acad Child Adolesc Psychiatry. 2010;49(10):980-9.

4. Kessler RC, Avenevoli S, Costello EJ, Georgiades K, Green JG, Gruber MJ, et al. Prevalence, persistence, and sociodemographic correlates of DSM-IV disorders in the National Comorbidity Survey Replication Adolescent Supplement. Arch Gen Psychiatry. 2012;69(4):372-80.

5. Kessler RC, Avenevoli S, Costello J, Green JG, Gruber MJ, McLaughlin KA, et al. Severity of 12-month DSM-IV disorders in the National Comorbidity Survey Replication Adolescent 
Supplement. Arch Gen Psychiatry. 2012;69(4):381-9. doi:10.1001/ archgenpsychiatry.2011.1603.

6. Foley DL, Goldston DB, Costello EJ, Angold A. Proximal psychiatric risk factors for suicidality in youth: the Great Smoky Mountains Study. Arch Gen Psychiatry. 2006;63(9):1017-24.

7. Pine DS, Cohen P, Gurley D, Brook J, Ma Y. The risk for earlyadulthood anxiety and depressive disorders in adolescents with anxiety and depressive disorders. Arch Gen Psychiatry. 1998;55(1):56-64.

8. Beesdo-Baum K, Pine D, Lieb R, Wittchen H. American College of Neuropsychopharmacology 51st Annual Meeting; Hollywood, Florida 2012.

9. Britton JC, Grillon C, Lissek S, Norcross MA, Szuhany KL, Chen $\mathrm{G}$, et al. Response to learned threat: An FMRI study in adolescent and adult anxiety. Am J Psychiatr. 2013;170(10):1195-204.

10. Strawn JR, Bitter SM, Weber WA, Chu WJ, Whitsel RM, Adler C, et al. Neurocircuitry of generalized anxiety disorder in adolescents: a pilot functional neuroimaging and functional connectivity study. Depress Anxiety. 2012;29(11):939-47.

11. Blackford JU, Pine DS. Neural substrates of childhood anxiety disorders: a review of neuroimaging findings. Child Adolesc Psychiatr Clin N Am. 2012;21(3):501-25.

12. Strawn JR, Wehry AM, DelBello MP, Rynn MA, Strakowski S. Establishing the neurobiologic basis of treatment in children and adolescents with generalized anxiety disorder. Depress Anxiety. 2012;29(4):328-39.

13. Monk CS, Klein RG, Telzer EH, Schroth EA, Mannuzza S, Moulton 3rd JL, et al. Amygdala and nucleus accumbens activation to emotional facial expressions in children and adolescents at risk for major depression. Am J Psychiatr. 2008;165(1):90-8. doi:10. 1176/appi.ajp.2007.06111917.

14. McClure EB, Monk CS, Nelson EE, Parrish JM, Adler A, Blair RJ, et al. Abnormal attention modulation of fear circuit function in pediatric generalized anxiety disorder. Arch Gen Psychiatry. 2007;64(1):97-106. doi:10.1001/archpsyc.64.1.97.

15. Roy AK, Fudge JL, Kelly C, Perry JS, Daniele T, Carlisi C, et al. Intrinsic functional connectivity of amygdala-based networks in adolescent generalized anxiety disorder. J Am Acad Child Adolesc Psychiatry. 2013;52(3):290-9 e2. doi:10.1016/j.jaac. 2012.12.010. This is a large study examining the functional neurophysiology of amygdala-based netoworks in a cohort of adolescents with GAD, and demonstrating dysfunctional, anxietyrelated connectivity between the amygdala and the medial prefrontal cortex, insula, and cerebellum.

16. Britton JC, Lissek S, Grillon C, Norcross MA, Pine DS. Development of anxiety: the role of threat appraisal and fear learning. Depress Anxiety. 2011;28(1):5-17. doi:10.1002/da. 20733.

17. Monk CS, Nelson EE, McClure EB, Mogg K, Bradley BP, Leibenluft $\mathrm{E}$, et al. Ventrolateral prefrontal cortex activation and attentional bias in response to angry faces in adolescents with generalized anxiety disorder. Am J Psychiatr. 2006;163(6):10917. doi:10.1176/appi.ajp.163.6.1091.

18. Monk CS, Telzer EH, Mogg K, Bradley BP, Mai X, Louro HM, et al. Amygdala and ventrolateral prefrontal cortex activation to masked angry faces in children and adolescents with generalized anxiety disorder. Arch Gen Psychiatry. 2008;65(5):568-76. doi:10. 1001/archpsyc.65.5.568.

19. Maslowsky J, Mogg K, Bradley BP, McClure-Tone E, Ernst M, Pine DS, et al. A preliminary investigation of neural correlates of treatment in adolescents with generalized anxiety disorder. J Child Adolesc Psychopharmacol. 2010;20(2):105-11. doi:10.1089/cap. 2009.0049.

20. Shackman AJ, Salomons TV, Slagter HA, Fox AS, Winter JJ, Davidson RJ. The integration of negative affect, pain and cognitive control in the cingulate cortex. Nat Rev Neurosci. 2011;12(3):154-
67. doi:10.1038/nrn2994. This article reviews the key functions of the anterior cingulate cortex with regard to emotional and cognitive processing.

21. Allman JM, Hakeem A, Erwin JM, Nimchinsky E, Hof P. The anterior cingulate cortex. The evolution of an interface between emotion and cognition. Ann N Y Acad Sci. 2001;935:107-17.

22. Strawn JR, Chu WJ, Whitsel RM, Weber WA, Norris MM, Adler $\mathrm{CM}$, et al. A pilot study of anterior cingulate cortex neurochemistry in adolescents with generalized anxiety disorder. Neuropsychobiology. 2013;67(4):224-9. doi:10.1159/000347090.

23. Milham MP, Nugent AC, Drevets WC, Dickstein DP, Leibenluft E, Ernst M, et al. Selective reduction in amygdala volume in pediatric anxiety disorders: a voxel-based morphometry investigation. Biol Psychiatry. 2005;57(9):961-6. doi:10.1016/j.biopsych.2005.01. 038.

24. De Bellis MD, Casey BJ, Dahl RE, Birmaher B, Williamson DE, Thomas KM, et al. A pilot study of amygdala volumes in pediatric generalized anxiety disorder. Biol Psychiatry. 2000;48(1):51-7.

25. Strawn JR, Wehry AM, Chu WJ, Adler CM, Eliassen JC, Cerullo MA, et al. Neuroanatomic abnormalities in adolescents with generalized anxiety disorder: a voxel-based morphometry study. Depress Anxiety. 2013;30(9):842-8. doi:10.1002/da.22089.

26. Liao M, Yang F, Zhang Y, He Z, Su L, Li L. White matter abnormalities in adolescents with generalized anxiety disorder: a diffusion tensor imaging study. BMC Psychiatry. 2014;14:41. doi: 10.1186/1471-244X-14-41.

27. Guyer AE, Choate VR, Detloff A, Benson B, Nelson EE, PerezEdgar K, et al. Striatal functional alteration during incentive anticipation in pediatric anxiety disorders. Am J Psychiatr. 2012;169(2): 205-12.

28. Guyer AE, Lau JY, McClure-Tone EB, Parrish J, Shiffrin ND, Reynolds RC, et al. Amygdala and ventrolateral prefrontal cortex function during anticipated peer evaluation in pediatric social anxiety. Arch Gen Psychiatry. 2008;65(11):1303-12. doi:10.1001/ archpsyc.65.11.1303.

29. Killgore WD, Yurgelun-Todd DA. Social anxiety predicts amygdala activation in adolescents viewing fearful faces. Neuroreport. 2005;16(15):1671-5.

30. Price RB, Siegle GJ, Silk JS, Ladouceur CD, McFarland A, Dahl $\mathrm{RE}$, et al. Looking under the hood of the dot-probe task: an fMRI study in anxious youth. Depress Anxiety. 2014;31(3):178-87. doi: 10.1002/da.22255.

31. Fitzgerald KD, Liu Y, Stern ER, Welsh RC, Hanna GL, Monk CS, et al. Reduced error-related activation of dorsolateral prefrontal cortex across pediatric anxiety disorders. J Am Acad Child Adolesc Psychiatry. 2013;52(11):1183-91.

32. Beesdo K, Lau JY, Guyer AE, McClure-Tone EB, Monk CS, Nelson EE, et al. Common and distinct amygdala-function perturbations in depressed vs anxious adolescents. Arch Gen Psychiatry. 2009;66(3):275-85. doi:10.1001/archgenpsychiatry.2008.545.

33. Strawn JR, Cotton S, Luberto LCM, Patino LR, Stahl LA, Weber WA, et al. Neurofunctional changes associated with mindfulnessbased cognitive therapy in anxious adolescents at risk for developing bipolar disorder. San Diego: American Academy of Child and Adolescent Psychiaty; 2014.

34. Mueller SC, Aouidad A, Gorodetsky E, Goldman D, Pine DS, Ernst M. Gray matter volume in adolescent anxiety: an impact of the brain-derived neurotrophic factor Val(66)Met polymorphism? J Am Acad Child Adolesc Psychiatry. 2013;52(2):184-95.

35. Strawn JR, Hamm L, Fitzgerald DA, Monk CS, Phan L. Neurostructural abnormalities in pediatric anxiety disorders. J Anxiety Disord. 2014; in revision.

36. Wehry AM, Eliassen J, Cerullo MC, Adler CM, Strakowski SM, DelBello MP et al. The neurostructural impact of co-occurring anxiety in pediatric patients with major depressive disorder: a voxel-based morphometry study. 34th Annual Meeting of the 
Anxiety and Depression Association of America, 2014; Chicago 2014.

37. Clauss JA, Blackford JU. Behavioral inhibition and risk for developing social anxiety disorder: a meta-analytic study. J Am Acad Child Adolesc Psychiatry. 2012;51(10):1066-75.e1. doi:10.1016/j. jaac.2012.08.002.

38. Blackford JU, Allen AH, Cowan RL, Avery SN. Amygdala and hippocampus fail to habituate to faces in individuals with an inhibited temperament. Soc Cogn Affect Neurosci. 2013;8(2):143-50. This study provides a unique insight into the neurophysiology of behavioral differences in children with inhibited temperament who are at risk for developing anxiety disorders.

39. Blackford JU, Avery SN, Cowan RL, Shelton RC, Zald DH. Sustained amygdala response to both novel and newly familiar faces characterizes inhibited temperament. Soc Cogn Affect Neurosci. 2011;6(5):621-9. doi:10.1093/scan/nsq073.
40. Pérez-Edgar K, Roberson-Nay R, Hardin MG, Poeth K, Guyer AE, Nelson EE, et al. Attention alters neural responses to evocative faces in behaviorally inhibited adolescents. Neuroimaging. 2007;35(4):1538-46. doi:10.1016/j.neuroimage.2007.02.006.

41. Hardee JE, Benson BE, Bar-Haim Y, Mogg K, Bradley BP, Chen G, et al. Patterns of neural connectivity during an attention bias task moderate associations between early childhood temperament and internalizing symptoms in young adulthood. Biol Psychiatry. 2013;74(4):273-9. doi:10.1016/j.biopsych.2013.01.036.

42. Telzer EH, Mogg K, Bradley BP, Mai X, Ernst M, Pine DS, et al. Relationship between trait anxiety, prefrontal cortex, and attention bias to angry faces in children and adolescents. Biol Psychol. 2008;79(2):216-22. doi:10.1016/j.biopsycho.2008.05.004.

43. Jarcho JM, Fox NA, Pine DS, Leibenluft E, Shechner T, Degnan $\mathrm{KA}$, et al. Enduring influence of early temperament on neural mechanisms mediating attention-emotion conflict in adults. Depress Anxiety. 2014;31(1):53-62. doi:10.1002/da.22140. 